\title{
Tracking and fusion for wireless sensor networks *
}

\author{
M. L. Hernandez, A. D. Marrs, S. Maskell, \\ QinetiQ Ltd, \\ Malvern Technology Centre, \\ Great Malvern, \\ UK, WR14 3PS \\ mlhernandez, admarrs, srmaskell @QinetiQ.com
}

\author{
M. R. Orton \\ Cambridge University Engineering Department, \\ Trumpington Street \\ Cambridge \\ UK, CB2 1PZ \\ mro20@eng.cam.ac.uk
}

\begin{abstract}
The recent interest in the development of wireless sensor networks for surveillance introduces new problems that will need to be addressed when developing target tracking algorithms for use in such networks. Specifically the power and stealth requirements when combined with the wireless communications architecture will lead to potentially significant delays in the measurement collection process.

The recent development of out-of-sequence tracking algorithms and posterior Cramér-Rao lower bounds for tracking with measurement origin uncertainty makes it possible to investigate how robust these new tracking algorithms are to a wide range of communications delays and a range of false alarm densities. This paper brings together these various components and presents the performance analysis for a simulated wireless network. Results show that position estimate accuracy close to the lower bound should be possible for communications intervals up to $4 \mathrm{~s}$ for challenging false alarm densities.
\end{abstract}

Keywords: tracking, sensor networks, out-of-sequence measurements, Cramér-Rao lower bound.

\section{Introduction}

\subsection{Background}

In the last 2-3 years there has been an increasing level of interest in the use of unattended ground sensors (UGS) or smart sensor nodes as components of a "Smart Sensor Web" (see [1][2] for examples).

It is envisaged that such sensor nodes will be networked together in an ad-hoc manner and be able to cope with dynamic changes in configuration of the sensor network.

Although there has been a significant amount of work on the development of sensor systems and the associated networking protocols for wireless communication in networks of such systems [3], much less effort has been expended in examining how tracking and data fusion algorithms can be adapted to deal with the unique processing problems such networks would present.

\footnotetext{
${ }^{*}$ Copyright QinetiQ Ltd 2002
}

The requirements for such wireless sensor networks to be energy efficient and, in a military domain, stealthy, places demands upon the communications protocols that will have a direct impact upon the ability to form target tracks from the observations made by the sensors in the network. For example, intermediate nodes in the network will be nominated as "parents" to gather observations from their "children". Those gathered observations will then be passed further up the network chain either as observation constructed from the raw sensor signal or as processed tracks formed from the local observations. Implicit in this collection process are the delays introduced as the "parents" communicate with their "children" in time sequence during the intervals when it is not acting as a "child" on another subnetwork and communicating with its "parent".

The delays introduced by the network protocols will result in out of sequence observations arriving at the "parent" node that has been nominated to form tracks. Recently, work within the tracking community has sought to develop tracking filters that deal with out-of-sequence measurements (OOSM) without recourse to "winding back" the filter, for example [4][5] [6][7] [8] deal with linear dynamic models and linear/non-linear measurement models in the form of the Kalman filter (KF) and extended Kalman filter $(\mathrm{EKF})$. More recently interest has focused upon nonlinear/non-Gaussian models in the form of the particle filter with the general non-linear OOSM particle filter of [9] and the approximately non-linear OOSM particle filter of [10].

The existence of such algorithms for tracking with OOSM, when applied to the area of tracking in wireless sensor networks, enables us to pose the question: how much delay can a tracking algorithm deal with and still give acceptable results? The work presented in this paper represents (to our knowledge) the first attempt to provide an answer to that question.

\subsection{Overview}

The outline of this paper is as follows, the next section details the development of a model for a networked sensor array. This is followed in section 3 by an overview of the state 
space model and particle filter that will be used to develop the tracking algorithm. A test scenario and corresponding results are then presented in section 4. Finally, the paper concludes with a discussion of the results in section 5 .

\section{Wireless network model}

\subsection{Background}

For a number of sensor nodes to be able to function in proximity to each other in conditions where they may belong to different communications nets a communications system that enables the nets to communicate without interfering with each other is required. This wireless network communication problem for ad-hoc networks has already been considered during the development of the Bluetooth architecture (see [11] for a good overview). In that case a frequency hopping Time Division Multiple Access (TDMA) approach was taken.

In such a frequency hopping Time Division Multiple Access (TDMA) system the nodes form piconets where one of the nodes becomes the parent (master in Bluetooth terminology) and the other $N_{s}$ nodes the children (slaves). Master/slave communication is then conducted by time slicing where the master broadcasts and the slaves receive during odd time slots and the master receives while the slaves broadcast during even time slots. Intra piconet collisions are avoided by only permitting one slave to communicate with the master during one time slot. This leads to the concept of the master polling a slave which then replies in the following time slice. This implies that a slave must wait for some number of time slots before being able to communicate with its master again.

This TDMA approach introduces a delay in communication while a slave waits for the piconet master to poll it. To minimise this delay, piconets are restricted in size. For example, with Bluetooth the number of slaves is limited to 7.

Piconets can be networked together by forming their master nodes into a net with one node being nominated as the master and the others becoming slaves for this net. In this manner a field of nodes could be "netted" together to form a tree architecture and all nodes can eventually communicate their messages to the global master node.

To model the effect of such a communications protocol we abstract the main features affecting the communications delay, namely the time duration between time slots in the TDMA architecture. For example, in the Bluetooth system a time slot has a duration of $625 \mu$ s with a packet size that can be either 1, 3 or 5 slots long. This means that the maximum duration for packet transfer between nodes is $3.125 \mathrm{~ms}$ and the maximum revisit interval for a slave in a full piconet might be $43.75 \mathrm{~ms}$ (assuming a maximum 5 slot packet being sent in each direction, sequential polling of the slaves by the master and no collisions with other neighbouring pi- conets).

Bluetooth was designed to replace the cables commonly required to connect modern IT systems together. As such, although it was designed with a low power requirement in mind, it was not designed with the specific power requirements that are characteristic of long term use away from battery recharging facilities. Nor was stealth a specific requirement. In an operational wireless sensor network, utilised as a surveillance asset in a military domain, network operation may be required to cover a period of weeks or even months. One way to conserve power and improve stealthiness, would be to extend the time interval between transfer of data packets between nodes. For example, in the case where the targets of interest are people, with a speed of $3-4 \mathrm{~m} / \mathrm{s}$, observations may only need to be made every $1 \mathrm{~s}$. In this case the $43.75 \mathrm{~ms}$ revisit interval would be overkill.

To investigate the potential for extending this time interval, we model the Bluetooth piconet architecture but make the time slot a user adjustable parameter. Conceptually we assume the communications to be carried out using the same $625 \mu$ s time slots but add a period of communications down time between master/slave transmission pairs. Thus we may have sensor nodes making observations every $1 \mathrm{~s}$, caching those observations to a buffer to await transmission to the master, which may occur on a master/slave transmission time interval of $1 \mathrm{~s}, 2 \mathrm{~s}, 3 \mathrm{~s}$ etc.

\section{Tracking algorithm}

\subsection{State space model}

Target tracking using sequential Bayesian estimation has a long history in the research literature (see [12][13] for reviews). In particular much work has focused upon the linear Gaussian state space model represented by the KF and its approximation to non-linear Gaussian models the EKF. Recently, there has been a rise in interest in the particle filter approximation to non-linear/non-Gaussian models [14][15]. Here we concentrate upon the particle filter approximation and in particular the OOSM version presented in [9].

Consider a target whose state from time $t_{k-1}$ to time $t_{k}$ evolves according to

$$
\mathbf{x}_{k}=f_{k, k-1}\left(\mathbf{x}_{k-1}\right)+\omega_{k, k-1}
$$

where $f_{k, k-1}(\cdot)$ is the state transition function and $\omega_{k, k-1}$ is the cumulative effect of the, possibly non-Gaussian, process noise in the interval $k-1$ to $k$. Indirect measurements of this evolving target state are made according to the equation

$$
\mathbf{z}_{k}=h_{k}\left(\mathbf{x}_{k}\right)+\epsilon_{k},
$$

where $h_{k}(\cdot)$ is a, possibly time varying, non-linear function of the underlying target state and $\epsilon_{k}$ is the measurement noise, which is drawn from an independent identically distributed (iid) white noise process. 
In the context of recursive Bayesian estimation, equation (1) can be used to define a prior density for the state at time $t_{k}, p\left(\mathbf{x}_{k} \mid \mathbf{x}_{k-1}\right)$, while equation (2) defines a likelihood function $p\left(\mathbf{z}_{k} \mid \mathbf{x}_{k}\right)$. When combined with Bayes' theorem a recursive formula for the posterior joint probability of the set of target states up to time $k, \mathbf{X}_{0: k}=\left\{\mathbf{x}_{i}: i \in\right.$ $0,1, \ldots, k\}$, given the set of measurements up to that time, $\mathbf{Z}_{1: k}=\left\{\mathbf{z}_{i}: i \in 1,2, \ldots, k\right\}$, can be written

$$
p\left(\mathbf{X}_{0: k} \mid \mathbf{Z}_{1: k}\right)=p\left(\mathbf{X}_{0: k-1} \mid \mathbf{Z}_{1: k-1}\right) \frac{p\left(\mathbf{z}_{k} \mid \mathbf{x}_{k}\right) p\left(\mathbf{x}_{k} \mid \mathbf{x}_{k-1}\right)}{p\left(\mathbf{z}_{k} \mid \mathbf{Z}_{1: k-1}\right)} .
$$

Exploiting the Markov property of the state evolution process, the marginal distribution for the target state at time $k, p\left(\mathbf{x}_{k} \mid \mathbf{Z}_{1: k}\right)$ can also be written recursively in terms of a prediction equation,

$$
p\left(\mathbf{x}_{k} \mid \mathbf{Z}_{1: k-1}\right)=\int p\left(\mathbf{x}_{k} \mid \mathbf{x}_{k-1}\right) p\left(\mathbf{x}_{k-1} \mid \mathbf{Z}_{1: k-1}\right) d \mathbf{x}_{k-1},
$$

and an update equation

$$
p\left(\mathbf{x}_{k} \mid \mathbf{Z}_{1: k}\right)=\frac{p\left(\mathbf{z}_{k} \mid \mathbf{x}_{k}\right) p\left(\mathbf{x}_{k} \mid \mathbf{Z}_{1: k-1}\right)}{\int p\left(\mathbf{z}_{k} \mid \mathbf{x}_{k}\right) p\left(\mathbf{x}_{k} \mid \mathbf{Z}_{1: k-1}\right) d \mathbf{x}_{k}} .
$$

The integrals in equations (4) and (5) cannot in general be computed analytically, rendering an analytic approach to computing the state posterior probability density function (pdf) infeasible except in special cases such as the linear Gaussian state space model (Kalman filter) and finite state space Hidden Markov model (HMM).

The applicability of the linear Gaussian state space model can be extended to nonlinear models by replacing the nonlinear models in the state evolution and measurement equations with a linearised approximation (the EKF).

Alternatively the probability density functions can be approximated, as in the particle filter.

\subsection{The particle filter}

The linearising approximation of the evolution and measurement models about the current state estimate leads to the main weakness of the EKF, namely if the system is sufficiently non-linear and non-Gaussian the linearised approximation leads to a poor prediction of the state sufficient statistics and subsequent measurement update. This then fails to provide a complete correction to those sufficient statistics. In particular, if the predicted state density is multimodal, this can result in a catastrophic loss of track.

Recent advances in nonlinear filtering [16] have seen the increasing utilisation of sample based approximations to the state pdf. We review the main points regarding such sample based approximations here.

Samples from the posterior density $p\left(\mathbf{X}_{0: K} \mid \mathbf{Z}_{1: K}\right)$ enable the pointwise evaluation of the prior density $p\left(\mathbf{x}_{k+1} \mid \mathbf{x}_{k}\right)$ and the likelihood $p\left(\mathbf{z}_{k+1} \mid \mathbf{x}_{k+1}\right)$. In general, the drawing of direct samples from $p\left(\mathbf{X}_{0: K} \mid \mathbf{Z}_{1: K}\right)$ is impossible, however, through the use of importance sampling [17] samples are drawn independently from an importance function $\pi\left(\mathbf{X}_{0: K} \mid \mathbf{Z}_{1: K}\right)$ with the same support as the posterior.

For recursive estimation, an importance function of the form

$$
\pi\left(\mathbf{X}_{0: K} \mid \mathbf{Z}_{1: K}\right)=\pi\left(\mathbf{x}_{0} \mid \mathbf{z}_{0}\right) \prod_{k=1}^{K} \pi\left(\mathbf{x}_{k} \mid \mathbf{X}_{0: k-1}, \mathbf{Z}_{1: k}\right)
$$

permits recursive evaluation of the un-normalised importance weights $w_{k}^{* i}$ as measurements $\mathbf{z}_{k}$ become available:

$$
w_{k}^{* i}=w_{k-1}^{* i} \frac{p\left(\mathbf{z}_{k} \mid \mathbf{x}_{k}^{i}\right) p\left(\mathbf{x}_{k}^{i} \mid \mathbf{x}_{k-1}^{i}\right)}{\pi\left(\mathbf{x}_{k}^{i} \mid \mathbf{X}_{0: k-1}^{i}, \mathbf{Z}_{1: k}\right)} .
$$

This defines the basic sequential importance sampling (SIS) filter for which the prior, $p\left(\mathbf{x}_{k}^{i} \mid \mathbf{x}_{k-1}^{i}\right)$, is often used as a suboptimal but tractable importance function.

The basic SIS filter is prone to degeneracy since, with importance functions of the form (7), the variance of the importance weights can only increase over time [15]. Such degeneracy can be limited by the use of the optimal importance function $p\left(\mathbf{x}_{k} \mid \mathbf{x}_{k-1}^{i}, \mathbf{z}_{k}\right)$, which minimises the variance of the importance weights. However, drawing from this optimal importance function is only possible in a few special cases. In general, for most system evolution and measurement models, a suboptimal but tractable importance function is used, taking the form of a convenient tractable function such as $p\left(\mathbf{x}_{k} \mid \mathbf{x}_{k-1}^{i}\right)$ which approximates the optimal importance function $p\left(\mathbf{x}_{k} \mid \mathbf{x}_{k-1}^{i}, \mathbf{z}_{k}\right)$. Markov chain Monte Carlo (MCMC) is often used to improve upon this approximation [18].

\subsection{Extension to out-of-sequence measure- ments}

The extension of the particle filter to deal with OOSM as presented in [9], are now summarised. At time $t_{k-1}$ we have an ordered set of in-sequence measurements $\mathbf{Z}_{1: k-1}$. The $k$ th measurement arrives and is found to be out-ofsequence such that $t_{k}<t_{k-1}$. If $b$ and $a$ are the time indices $\boldsymbol{b}$ efore and $\boldsymbol{a}$ fter the OOSM, such that $t_{b}<t_{k}<t_{a}$ and $a=b+1$ in the order sequence $\mathbf{Z}_{1: k-1}$, the updated posterior is

$$
\begin{gathered}
p\left(\mathbf{X}_{0: k} \mid \mathbf{Z}_{1: k}\right)=p\left(\mathbf{X}_{0: b} \mid \mathbf{Z}_{1: b}\right) \times \frac{p\left(\mathbf{z}_{k} \mid \mathbf{x}_{k}\right) p\left(\mathbf{x}_{k} \mid \mathbf{x}_{b}\right) p\left(\mathbf{x}_{a} \mid \mathbf{x}_{k}\right)}{p\left(\mathbf{z}_{k}\right)} \times \\
p\left(\mathbf{X}_{a: k-1} \mid \mathbf{Z}_{a: k-1}\right)
\end{gathered}
$$

which can be simplified to

$$
\begin{aligned}
p\left(\mathbf{X}_{0: k} \mid \mathbf{Z}_{1: k}\right)= & p\left(\mathbf{X}_{0: k-1} \mid \mathbf{Z}_{1: k-1}\right) \times \\
& \frac{p\left(\mathbf{x}_{k}^{i} \mid \mathbf{x}_{b}^{i}\right) p\left(\mathbf{x}_{a}^{i} \mid \mathbf{x}_{k}^{i}\right) p\left(\mathbf{z}_{k} \mid \mathbf{x}_{k}^{i}\right)}{p\left(\mathbf{x}_{a}^{i} \mid \mathbf{x}_{b}^{i}\right) p\left(\mathbf{z}_{k}\right)}
\end{aligned}
$$


Following the methodology of importance sampling, we may now write down the equation for the un-normalised importance weights

$$
w_{k}^{* i}=w_{k-1}^{* i} \frac{p\left(\mathbf{z}_{k} \mid \mathbf{x}_{k}^{i}\right) p\left(\mathbf{x}_{k}^{i} \mid \mathbf{x}_{b}^{i}, \mathbf{x}_{a}^{i}\right)}{\pi\left(\mathbf{x}_{k}^{i} \mid \mathbf{X}_{0: k-1}^{i}, \mathbf{Z}_{1: k}\right)} .
$$

The choice of importance function is critical to the efficient operation of the particle filter. In [15] the optimal importance function is defined as that which minimises the variance of the importance weights conditional upon the simulated trajectory $\mathbf{X}_{0: k-1}^{i}$ and the observations $\mathbf{Z}_{1: k}$. It can be shown that $\pi\left(\mathbf{x}_{k}^{i} \mid \mathbf{X}_{0: k-1}^{i}, \mathbf{Z}_{1: k}\right)=p\left(\mathbf{x}_{k}^{i} \mid \mathbf{x}_{a}^{i}, \mathbf{x}_{b}^{i}, \mathbf{z}_{k}\right)$ is the importance function which minimises the variance of the importance weights conditional upon $\mathbf{X}_{0: k-1}^{i}$ and $\mathbf{Z}_{1: k}$.

Again this choice of importance function is not tractable but a tractable approximation is $p\left(\mathbf{x}_{k} \mid \mathbf{x}_{b}^{i}, \mathbf{x}_{a}^{i}\right)$ and with this choice of importance function the weights are given by $w_{k}^{* i}=w_{k-1}^{* i} p\left(\mathbf{z}_{k} \mid \mathbf{x}_{k}^{i}\right)$.

Drawing samples from this optimal importance function in many cases is not tractable. However, in the case of linear Gaussian dynamics, the optimal importance sampling function is available for sampling and is given by a Gaussian [15], with state dynamics of the form

$$
\mathbf{x}_{k+1}=F_{k, k-1} \mathbf{x}_{k}+\mathbf{Q}_{k, k-1}^{1 / 2} \mathbf{u}_{k}
$$

where $F_{k, k-1}$ is the state transition matrix from time $t_{k-1}$ to time $t_{k}, \mathbf{Q}_{k, k-1}$ is the corresponding covariance matrix for the transition and $\mathbf{u}_{k}$ is a white noise vector. Combining the priors $p\left(\mathbf{x}_{k} \mid \mathbf{x}_{b}\right)$ and $p\left(\mathbf{x}_{a} \mid \mathbf{x}_{k}\right)$ a sample $\mathbf{x}_{k}^{i}$ from $p\left(\mathbf{x}_{o}^{i} \mid \mathbf{x}_{b}^{i}, \mathbf{x}_{a}^{i}\right)$ can be generated from the equation

$$
\mathbf{x}_{k}^{i}=F_{B} \mathbf{x}_{b}+F_{A} \mathbf{x}_{a}+\mathbf{Q}_{k}^{1 / 2} \mathbf{u}_{k},
$$

where

$$
\begin{gathered}
\mathbf{Q}_{k}=\left(\mathbf{Q}_{k, b}^{-1}+F_{a, k}^{T} \mathbf{Q}_{a, k}^{-1} F_{a, k}\right)^{-1} \\
F_{B}=\mathbf{Q}_{k} \mathbf{Q}_{k, b}^{-1} F_{k, b} \\
F_{A}=\mathbf{Q}_{k} F_{a, k}^{T} \mathbf{Q}_{a, k}^{-1} .
\end{gathered}
$$

Computationally it is impractical to model $p\left(\mathbf{X}_{0: k} \mid \mathbf{Z}_{1: k}\right)$ so we model $p\left(\mathbf{X}_{k-L: k} \mid \mathbf{Z}_{k-L: k}\right)$ where $L$ is a predefined lag.

\subsection{Markov Chain Monte Carlo Smoothing}

To overcome the potential problem of degeneracy in the sequential importance sampling form of the OOSM particle filter, we introduce a MCMC smoothing step that enables the particles to efficiently explore the support of the state posterior. The use of a generic MCMC step, utilising the prior $p\left(\mathbf{x}_{k} \mid \mathbf{x}_{k-1}\right)$, proved to be particularly inefficient for the OOSM particle filter so an independence MCMC step was introduced.

The independence MCMC step essentially proposes the next state in the chain independent of the previous state and comprises a Gaussian proposal function $Q\left(\overline{\mathbf{X}}_{1: k}, \boldsymbol{\Sigma}_{1: k}\right)$ where

$$
\begin{gathered}
\overline{\mathbf{X}}_{1: k}=\frac{1}{N} \sum_{j=1}^{N} \mathbf{X}_{1: k}^{j}, \\
\boldsymbol{\Sigma}_{1: k}=\frac{1}{N-1} \sum_{j=1}^{N}\left(\overline{\mathbf{X}}_{1: k}-\mathbf{X}_{1: k}^{j}\right)\left(\overline{\mathbf{X}}_{1: k}-\mathbf{X}_{1: k}^{j}\right)^{T} .
\end{gathered}
$$

This function proposes moves that lie in the support of a Gaussian with the same mean and covariance as the particle histories up to time $k$ and enabled the MCMC chain to efficiently explore the state posterior.

Although the proposal is defined in terms of the whole particle history up to time $k$, to make the computation tractable when implementing it on a computer, the proposal is defined for a pre-determined finite lag. The choice of lag depends on the expected observation delay for the scenario of interest and any observations that have been delayed for a time greater than that represented by the chosen lag are automatically discarded.

\section{Simulation Results}

\subsection{Scenario}

For the simulated test scenario we model a small sensor network comprising three piconets formed into a scatternet. The master node for the scatternet represents the "command" node where data fusion and tracking will be performed. A schematic overview of the scenario is show in figure 1. The target motion is modelled as constant velocity $(\mathrm{CV})$ using the standard equation

$$
\mathbf{x}_{k}=F_{k, k-1}\left(\mathbf{x}_{k-1}\right)+\omega_{k, k-1}
$$

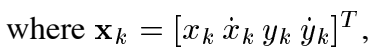

$$
F_{k, k-1}=\left[\begin{array}{cccc}
1 & T_{k} & 0 & 0 \\
0 & 1 & 0 & 0 \\
0 & 0 & 1 & T_{k} \\
0 & 0 & 0 & 1
\end{array}\right]
$$

and the process noise $\omega_{k, k-1}$ is white Gaussian with nonsingular covariance

$$
Q_{k, k-1}=q\left[\begin{array}{cccc}
\frac{T_{k}^{3}}{3} & \frac{T_{k}^{2}}{2} & 0 & 0 \\
\frac{T_{k}^{2}}{2} & T_{k} & 0 & 0 \\
0 & 0 & \frac{T_{k}^{3}}{3} & \frac{T_{k}^{2}}{2} \\
0 & 0 & \frac{T_{k}^{2}}{2} & T_{k}
\end{array}\right],
$$

where $q=0.1$. Although this represents a target that is not manoeuvering excessively, it provides a good benchmark to bracket the range of time intervals and false alarm rates where tracking is possible.

The observation rate for each of the sensor nodes in the scenario was fixed at $1 \mathrm{~s}$ and the scenario was re-run for a 
variety of communication rates $(\Delta \mathrm{T}=[1 s, 2 s, 3 s 4 s]$ to investigate the resulting distribution of delays and their effect on the tracking performance. The scenarios were also run for a variety of clutter densities to investigate their impact on performance when combined with the delays.

The sensors were modelled as simple bearings-only sensor types representative of an acoustic array. Their measurement noise standard deviation was set as $\sigma_{R}=1.0^{\circ}$. The initial target state was $x_{1}=[88.3-4.0766 .6-4.0]^{T}$.

To deal with the false alarm measurements in the filtering algorithm, the measurement likelihood was modified using the expected likelihood approach of [22].

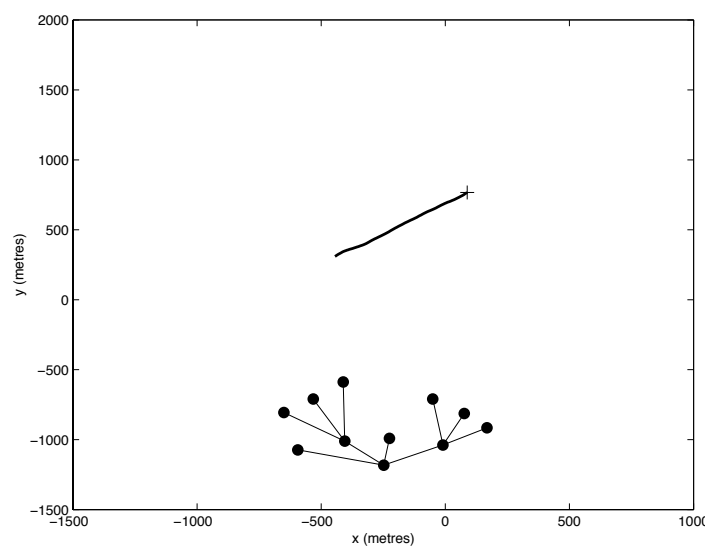

Figure 1: Scenario, target trajectory start denoted by the cross. The communication links within the scatternet are also drawn

\subsection{Results}

For the performance analysis of an algorithm to provide real insight, it is useful to be able to compare the results from the tracking algorithm with some benchmark. The generally accepted benchmark for tracking performance is the Posterior Cramér-Rao Lower Bound (pCRLB) [19]. Recently the expression for the pCRLB has been developed for tracking in clutter with either a linear measurement model [20] or a non-linear measurement model [21]. The ability to calculate such bounds open up for us the possibility of comparing our tracker's performance with the pCRLB for a range of communication intervals and false alarm densities.

False alarms were simulated in the standard manner [12] by modelling them as being uniformly distributed over the observation volume $V$ defined in terms of a validation gate

$$
V=2 * g * \sigma_{R},
$$

with the distribution of the number of false alarms $\left(m_{k}\right)$ being Poisson

$$
\mu\left(m_{k}\right)=\exp (-\lambda V) \frac{\left.(\lambda V)^{(} m_{k}\right)}{m_{k} !} .
$$

For our experiments we set the probability of detection to be $P_{D}=0.9$ and the range $\lambda=$ [0 1.4 2.8 4.25.7 7.18.5] per radian, which equates to an expected number of false alarms in the validation gate of $\bar{m}_{k}=$ [0 0.250 .50 .751 .01 .251 .5$]$ for a measurement standard deviation of $\sigma_{R}=1^{\circ}$ and a value of $g=5$. False alarms were modelled separately for each sensor thus $\bar{m}_{k}=1.5$ represents an expected false alarm rate at the tracking node of $1.5 * N_{S}$ or in our scenario with 11 sensors $\bar{m}_{k}{ }^{t}=16.5$ at the tracking node.

For each value of communication interval and false alarm density a number of Monte Carlo runs were performed using a state prior covariance of

$$
P_{1: 0}=\left[\begin{array}{cccc}
1000 & 0 & 0 & 0 \\
0 & 10 & 0 & 0 \\
0 & 0 & 1000 & 0 \\
0 & 0 & 0 & 10
\end{array}\right]
$$

For each run the particle filter was initialised to have a mean drawn from a Gaussian centered upon the true target state and having covariance of $P_{1: 0}$. The particle filter had $N=$ 1000 particles and the filter was limited to a lag of 60 .

An example of the distribution of delays occurring for each of the communication rates is shown in figure 2, while an example comparison of the RMS error in the target $x$ position estimate with the pCRLB for the whole trajectory is shown in figure 3.

The results for the complete ensemble of Monte Carlo runs are summarised by comparing the RMS error in the target position estimate at the end of the trajectory with the corresponding pCRLB. These RMS errors are shown as box plots in figures 4 and 5 while the pCRLBs for the case with no OOSM are shown as dashed lines. The box plots show the lower quartile, median and upper quartile as the bottom, middle and upper line of the box, the lines extending beyond the boxes show the extent of the rest of the sample with outliers show as a cross. The notch in the box gives an estimate of the mean. Time constraints restricted the Monte Carlo sample size to 20 thus exaggerating the effect of outliers resulting from a lost track. The plots show that as the delay increases the $x$ RMS error (which roughly equates to cross range in our scenario) shows only a small increase with the general trend following that of the pCRLB. For the $y$ RMS error (roughly equating to range in our scenario) the increase with increasing delay is more marked with the trend being slightly steeper than that of the pCRLB.

These results would indicate that there is significant scope for increasing the communications interval while maintaining acceptable tracking performance even in the presence of significant false targets.

\section{Conclusions}

An investigation into the tracking performance for a simulated wireless sensor network is presented. The unique 

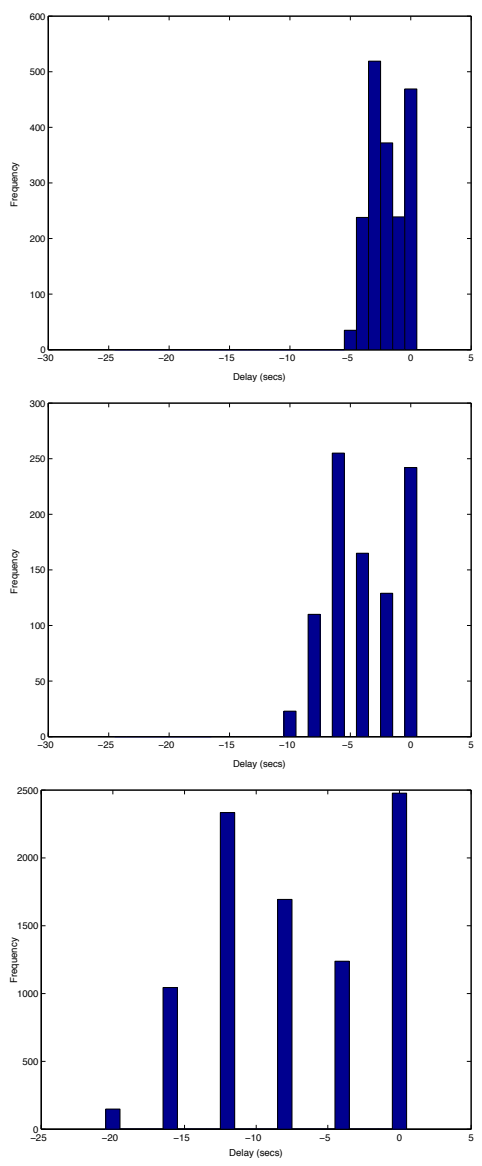

Figure 2: Histograms of delays experienced by observation packets for (from left to right) comms $\Delta \mathrm{T}=1.0 \mathrm{~s}, 2.0 \mathrm{~s}, 4.0 \mathrm{~s}$

communications architecture of such networks and the requirement for low power and stealthy operation will introduce delays into the process of collecting sensor measurements at the node designated to form target tracks.

The impact of these delays for a variety of communication intervals is assessed using the recently developed particle filter for tracking with out-of-sequence measurements. Evaluation of this assessment is aided by the use of the Posterior Cramér-Rao Lower Bound for non-linear measurement models and measurement origin uncertainty. Such an analysis has not been possible until the recent development of these tools.

Our results indicate that position estimate accuracy close to that predicted by the lower bound should be possible for communications intervals up to $4 \mathrm{~s}$ for challenging false alarm rates.

Future work will extend the analysis to include longer communication intervals, more maneuverable targets and investigate the impact of a multi-frame assignment approach to track-to-measurement association.

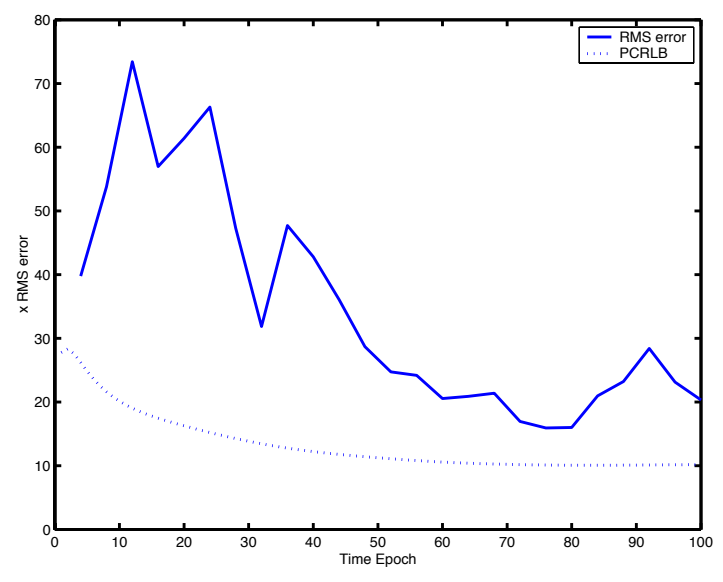

Figure 3: Example $x$ RMS error and pCRLB for comms $\Delta \mathrm{T}=4.0 \mathrm{~s}$
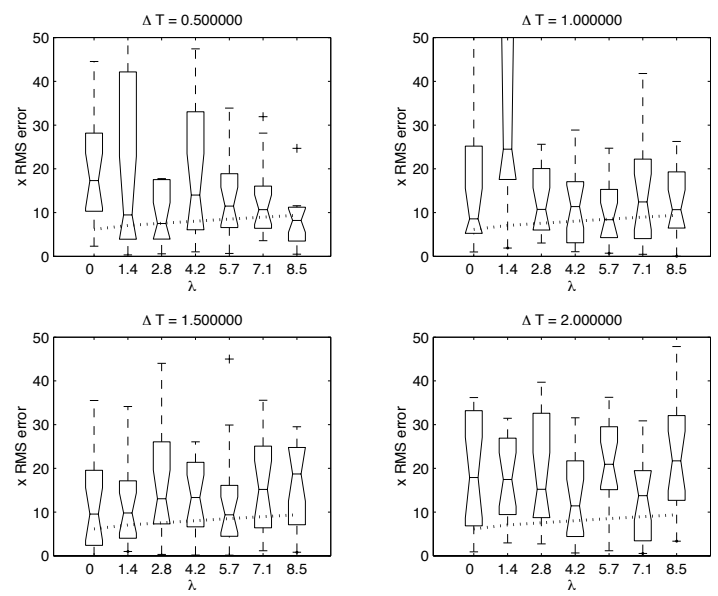

Figure 4: Comparison of $x$ RMS error and pCRLB (dotted line) at end of trajectory

\section{Acknowledgements}

This work was supported by the United Kingdom Ministry of Defence Corporate Research Programme CIP contract CRP11N/TG10.

\section{References}

[1] Pottie G. J., and W. J. Kaiser, "Wireless integrated network sensors", Communications of the ACM, Vol. 43, No. 5, May 2000.

[2] Lim A., "Architecture for dynamic information dissemination and fusion in distributed sensor networks", in: Proceedings of the 4th International conference on Information fusion, August 2001.

[3] Intanagonwiwat C., Govindan R., and D. Estrin, "Directed Diffusion: A Scalable and Robust Communica- 

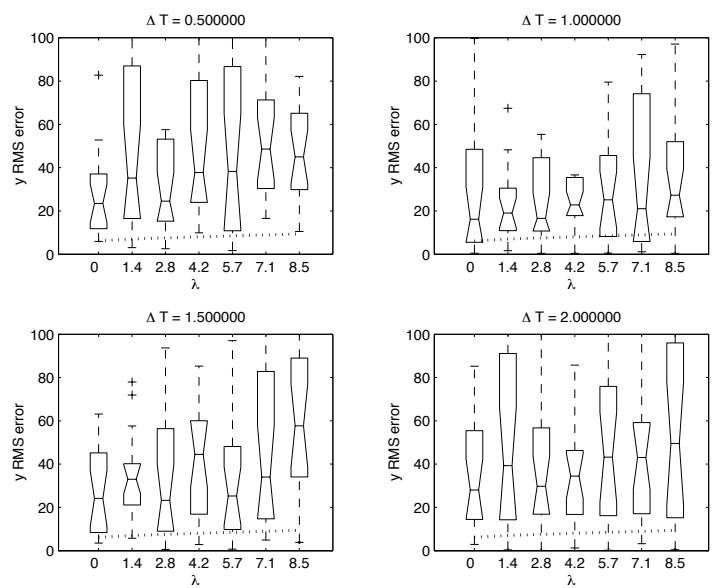

Figure 5: Comparison of $y$ RMS error and pCRLB (dotted line) at end of trajectory

tion Paradigm for Sensor Networks", ACM Mobicom, 2000.

[4] Hilton R. D., Martin D. A., and W. D. Blair, "Tracking with Time-Delayed Data in Multisensor Systems", NSWCDD/TR-93/351, Dalhgren, VA, August 1993.

[5] Bar-Shalom Y., "Update with Out-of-Sequence Measurements in Tracking: Exact Solution", in Signal and Data Processing of Small Targets: Proceedings of SPIE, vol. 4048, April 2000.

[6] Bar-Shalom Y., Mallick M., Chen H., and R. Washburn, "One-Step Solution for the General Out-of-Sequence Measurements Problem, in Proceedings 2001 IEEE Aerospace Conference, March 2001.

[7] Mallick M., Coraluppi S., and C. Carthel, "Advances in Asynchronous and Decentralised Estimation", in Proceedings 2001 IEEE Aerospace Conference, March 2001.

[8] Mallick M., Coraluppi S., and Y. Bar-Shalom, "Comparison of Out-of-Sequence Measurement Algorithms in Multi-platform Target Tracking", in Proceedings of the 4th International Conference on Information $\mathrm{Fu}$ sion, August 2001.

[9] Orton M. R., and Marrs A. D., "A Bayesian Approach to Multi-target Tracking and Data Fusion with Out-ofSequence Measurements", in Proceedings of the IEE Colloquium on Target Tracking: Algorithms \& Applications, October 2001.

[10] Mallick M., Kirubarajan T., and S. Arulampalam, "Out-of-Sequence Measurement Processing for Tracking Ground Targets Using Particle Filters ”, to appear in
Proceedings 2002 IEEE Aerospace Conference, March 2002.

[11] Bray J., Sturman C. F., "Bluetooth 1.1: Connect Without Cables", Prentice Hall PTR, ISBN 0-13-066106-6, 2002.

[12] Bar-Shalom Y., and X. Li, "Estimation and Tracking: Principles, Techniques and Software”, Artech House, 1993.

[13] Blackman S., and R. Popoli, "Design and Analysis of Modern Tracking Systems”, Artech House, 1999.

[14] Gordon N. J., Salmond D. J., and A. F. M. Smith, "Novel approach to nonlinear non-Gaussian Bayesian State Estimation", IEE Proceedings-F Radar, Sonar \& Navigation, vol. 140, APril 1993.

[15] Doucet A., Godsill S., and C. Andrieu, "On Sequential Monte Carlo Sampling Methods for Bayesian Filtering", Statistics and Computing, vol. 10, no. 3, 2000.

[16] Doucet A., deFreitas J. F. G., and N. J. Gordon, "Sequential Monte Carlo Methods in Practice”, Springer, 2001.

[17] Geweke J., "Bayesian Inference in Econometrics Models using Monte Carlo Integration”, Econometrica, vol. $57,1989$.

[18] Berzuini C., and W. R. Gilks, "Resample-move filtering with cross-model jumps", in Sequential Monte Carlo Methods in Practice, Springer, 2001.

[19] Tichavsky P., Muravchik C. H., and A. Nehorai, "Posterior Carmer-Rao Bounds for Discrete-Time Nonlinear Filtering", IEEE Transactions on Signal Processing, 46(5), pp. 1386-1396, 1998. 